\title{
Bedeutung der psychologischen Sicherheit für die Innovationsfähigkeit von Organisationen
}

\section{Entwicklung und Gestaltung von psychologischer Sicherheit in Unternehmen und Auswirkung auf deren Innovationsfähigkeit}

\author{
Bettina Hoffmann ${ }^{1,2}$. Dominik Hanisch ${ }^{3,4,5}$
}

Received: 23 July 2020 / Accepted: 18 February 2021 / Published online: 16 March 2021

(c) The Author(s) 2021

\begin{abstract}
The future of a company depends to a large extent on whether it can establish market-relevant innovations. In order to develop innovations and thus build sustainable business models, companies should operate their own innovation management. This not only systematically develops innovations, it also creates organizational framework conditions so that an innovative company culture can flourish better. But not only the organizational aspects lead to an increased ability to innovate, it is also the interpersonal interactions that make a decisive contribution. The quality and the result of human interaction depends in a decisive way on how safe people feel in the current group setting. The more confident they feel, the more the group members dare to ask questions, admit weaknesses and mistakes, contribute ideas and question the status quo - the foundations for innovation. If a company wants to develop the basis for psychological safety, then it is important to create suitable conditions in order to be able to make full use of the performance potential of all employees. Freedom, trust and participation are particularly important in innovation in order to promote creativity and the ability to innovate.
\end{abstract}

Keywords Psychological Safety $\cdot$ Innovation $\cdot$ Ability to innovate $\cdot$ Leadership

\section{Zusammenfassung}

Die Zukunft eines Unternehmens ist massgeblich davon abhängig, ob es marktrelevante Innovationen etablieren kann. Um Innovationen zu entwickeln und damit zukunftsfähige Geschäftsmodelle aufzubauen, sollten Unternehmen ein eigenes Innovationsmanagement betreiben. Dieses entwickelt nicht nur systematisch Innovationen, es schafft auch organisationale Rahmenbedingen, damit eine innovative Firmenkultur besser gedeihen kann. Doch nicht nur die organisationalen Aspekte führen zu einer erhöhten Innovationsfähigkeit, es sind auch die zwischenmenschlichen Interaktionen, die massgebend dazu beitragen. Die Qualität und das Ergebnis menschlicher Interaktion hängt in entscheidender Weise davon ab, wie sicher sich Menschen in dem aktuellen Gruppensetting fühlen. Je sicherer sie sich fühlen, um so eher trauen sich die Gruppenmitglieder Fragen zu stellen, Schwächen und Fehler zuzugeben, Ideen einzubringen sowie den Status quo zu hinterfragen - die Grundlagen für Innovation. Wenn ein Unternehmen die Basis für psychologische Sicherheit entwickeln möchte, dann ist es wichtig, geeignete Voraussetzungen zu schaffen um das Leistungspotenzial aller Mitarbeitenden umfassend nutzen zu können. Gerade in der Innovation sind Freiräume, Vertrauen und Partizipation besonders wichtig, um die Kreativität und die Innovationsfähigkeit zu fördern.

Schlüsselwörter Innovationsfähigkeit · Innovation · Psychologische Sicherheit · Leadership

Dominik Hanisch

d.hanisch@hawess.ch

1 Dozentin Universität St. Gallen \& Hochschule Für Wirtschaft in Zürich, St. Gallen, Switzerland

2 Geschäftsführerin B.HOFFMANN \& NETWORK, Netzwerkpartnerin Pfyffer Schmid, Zurich, Switzerland
3 Innovationsmanager bei Energie Wasser Bern, Bern, Switzerland

4 Dozent an der Hochschule für Wirtschaft in Zürich, Zurich, Switzerland

5 Gründer und Geschäftsführer von INNOVATIONSHELDEN \&, HAWESS SCHWEIZ GMBH, Bargen, Switzerland 


\section{Vorwort}

Grossangelegte Studien wie zum Beispiel der Bericht «Forschung und Innovation in der Schweiz» (2020) vom Staatssekretariat für Bildung, Forschung und Innovation SBFI thematisieren übergeordnet die Leistungsfähigkeit des Schweizer Forschungs- und Innovationssystems. Die jährliche Studie des Beratungsunternehmens The Boston Consulting Group fokussiert sich auf die Innovationsfähigkeit von Unternehmen. Und der Global-Innovation-Index vergleicht die Innovationskraft verschiedener Länder. Es gibt zahlreiche Studien, Berichte und Rankings zum Thema Innovation. Und die Schweiz schneidet praktisch überall mit Spitzenwerten ab. Jedoch ist sind häufig organisationale Aspekte deren Forschungsgegenstand und eher selten wird die Interaktion zwischen den Menschen erforscht. Dieser Artikel befasst sich mit der Gestaltung der zwischenmenschlichen Interaktionen und zeigt auf, wie sie einen wesentlichen Beitrag leisten kann, um die organisationale Innovationsfähigkeit zu erhöhen. Hierbei fokussiert dieser Artikel auf die von den Menschen wahrgenommene sogenannte psychologischen Sicherheit und zeigt auf, wie das Innovationsmanagement einen Beitrag dazu leisten kann.

\section{Ausgangslage}

Die Zukunft eines Unternehmens entscheidet sich massgeblich an ihrer Fähigkeit, marktrelevante Innovationen hervorzubringen. Dabei ist viel über den Innovationsprozess (vgl. Goffin et al. 2010) oder über das dazu notwendige Innovationsmanagement geschrieben worden (vgl. Hauschildt et al. 2016; Cooper 2009; Chesbrough 2006). Viele Innovationen sind das Ergebnis eines nicht planmässigen oder intendierten, sondern zufälligen Prozesses. Die meisten, vor allem grösseren Organisationen, versuchen Innovationen gezielt durch ein Innovationsmanagement zu fördern und Innovationen über eine Innovationsstrategie zu planen. Gleichzeitig wird aber der Interaktion der einzelnen am Innovationsprozess beteiligten Personen nach wie vor weniger Aufmerksamkeit geschenkt.

In diesem Artikel soll das wissenschaftliche Konstrukt der psychologischen Sicherheit von Amy Edmondson mit Innovationsfähigkeit einer Organisation in Verbindung gebracht werden. Die zentrale Frage lautet, wie wirkt sich das Erleben von psychologischer Sicherheit auf die Innovationsfähigkeit einer Organisation aus?

\section{Innovationsfähigkeit - organisationale und psychologische Aspekte}

Als Erfolgsfaktoren für eine hohe Innovationsfähigkeit werden immer wieder die Unternehmenskultur, eine klare Innovationsstrategie als auch eine Markt- und Kundenorientierung also weitgehend organisationale Aspekte benannt (vgl. Raabe 2012). Weiterhin wird aber auch in der Innovationsforschung erwähnt, dass die Innovationsfähigkeit eines Teams von gruppendynamischen Aspekten abhängt, also von der Frage, wie die einzelnen Mitglieder des Teams interagieren. Hier wird einerseits auf den positiven Einfluss einer offenen und transparenten Kommunikation im Allgemeinen hingewiesen-wobei selten genau ausgeführt wird, wie man eine offene und transparente Kommunikation beobachten kann. Andererseits wurde dem Aspekt des Lernens Aufmerksamkeit geschenkt. Kels, Baumann und Kocher haben in dieser Zeitschrift auf die positive Korrelation zwischen Lern- und Erneuerungsfähigkeit einer Organisation und Innovationsperformance hingewiesen (vgl. Kels et al. 2019, S. 125-129).

\section{Innovieren bedeutet den Status quo in Frage stellen}

Schumpeter prägte den Begriff «der schöpferischen Zerstörung» als Grundlage für Innovation, unternehmerisches Wachstum und Wohlstand. Auch wenn er in seinen Erörterungen eine volkswirtschaftliche Perspektive einnahm, wird deutlich, dass Innovation einen radikal anderen Blick auf das Gewohnte, Bekannte und Alte erfordert. Nur so entstehen neue Sichtweisen und Ideen. Gleichzeitig braucht es dafür Mut und Risikobereitschaft jeden einzelnen und einer Gruppe, sowohl etwas Bewährtes in Frage zu stellen als auch, etwas Neues auszuprobieren. Nun könnte man argumentieren, dass es ja gewissermassen einer institutionellen Ermutigung gleichkommt, wenn ein Team den Projektauftrag erhält, Ideen für Innovationen zu generieren. Das ist richtig und auch der Grund, warum systematisches Innovationsmanagement betrieben wird. Was in dieser Argumentation dann aber nicht berücksichtigt wird, sind die individualpsychologischen und gruppendynamischen Effekte innerhalb eines Teams. Auch wenn ein Team den Auftrag erhält, innovativ zu denken und Bewährtes in Frage zu stellen, stellt sich etwas in der Gruppe ein, dass Edgar Schein ersetzen durch Meyer et al. in seinen Arbeiten als Lernangst beschrieben hat (vgl. Meyer et al. 2018). Damit bezeichnet er die Angst, sich vor einer Gruppe als inkompetent zu erweisen, was zwei Effekte haben kann: Zum einen werden möglicherweise gute, spontane Ideen nicht benannt, was wiederum den gruppendynamischen Kreativitätsprozess bremst. Zum anderen führt aber Angst bei jedem 
individuell zur Begrenzung seiner Kreativität. Damit stellt sich die Frage, wie dieser Angst begegnet werden kann.

\section{Projekt Aristotle von Google}

Ein Grossteil der Arbeit wird in Teams erledigt. In diesen Einheiten werden innovative Ideen geboren, entwickelt und getestet. In ihnen verbringen die Mitarbeiter die meiste Zeit. Aber diese Teams sind auch die Orte, an denen zwischenmenschliche Probleme, schlechte Eigenschaften und unklare Gruppenziele zu Unproduktivität und Spannungen führen können.

Im Jahr 2012 startete Google eine Initiative mit dem Codenamen «Project Aristotle», um Hunderte von Google-Teams $\mathrm{zu}$ untersuchen und herauszufinden, warum einige gescheitert sind, während andere erfolgreich waren (vgl. New York Times Magazine 2016). Die Namenswahl bezieht sich auf das Zitat des Philosophen Aristoteles: „Das Ganze ist mehr als die Summe seiner Teile “. Das Projekt Aristoteles verlief über einen Zeitraum von zwei Jahren und startete mit der Hypothese, dass vor allem die Zusammensetzung eines Teams von entscheidender Bedeutung für ein erfolgreiches Team sein müsse.

Zunächst war es nötig zu definieren, was genau ein Team eigentlich ist. In Abgrenzung zu Arbeitsgruppen, deren Mitglieder nur zu einem geringen Teil voneinander abhängig sind, ist innerhalb eines Teams eine weitaus verflochtenere Struktur anzutreffen. Teams planen Aufgaben, lösen Probleme, treffen Entscheidungen und überprüfen den Projektfortschritt gemeinsam. Die Teammitglieder sind auf ihre Kollegen angewiesen und teilweise abhängig, um Aufgaben erledigen zu können.

Die untersuchten Teams - eine Mischung aus insgesamt 180 leistungsstarken und weniger effektiven Teams - wurden auf ihre Zusammensetzung und die Gruppendynamik hin analysiert. Dabei konnte festgestellt werden, dass die Effektivität eines Teams weniger von den Personen im Team abhing, als vielmehr von der Art und Weise, wie sie zusammenarbeiteten. Dabei identifizierten die Forscher in den leistungsstarken Teams immer wieder die gleichen Themen:

1. Effekt. Tragen die individuellen Arbeiten der Personen zum Gesamterfolg des Teams bei?

2. Sinn. Erkennen die Mitglieder Sinn in ihrer Arbeit? Der Sinn der Arbeit kann sich dabei individuell sehr stark unterscheiden: finanzielle Sicherheit, Unterstützung der Familie oder Selbstverwirklichung des Individuums.

3. Sicherheit. Wie sicher und dazugehörig fühlen sich die Mitglieder in ihrem Team? Vertrauen sie einander und nehmen sie sich gegenseitig ernst?

4. Zuverlässigkeit. Als wie zuverlässig schätzt sich das Team ein? Werden Arbeiten pünktlich erledigt?

5. Struktur. Wissen die einzelnen Teammitglieder welche Anforderungen wie erfüllt werden können? Kennen sie die kurz- und langfristigen Ziele, an welchen das Team arbeitet?

Die aktuelle Situation im eigenen Team zu erfassen, ist der erste Schritt in Richtung mehr Teameffektivität. Darauf aufbauend können dann Massnahmen ergriffen werden, um schließlich eine höhere psychologische Sicherheit zu erreichen.

\section{Das Konzept der psychologischen Sicherheit}

1999 veröffentlicht Amy Edmondson, Professorin an der Harvard Universität des von Novartis gesponserten Lehrstuhl für Leadership und Management, einen Beitrag über psychologische Sicherheit in Teams und ihre Auswirkungen auf das Lernen in Gruppen (vgl. Edmondson 1999, S. 350-383). Damit verbindet sie eine individuelle Sichtweise mit einer gruppendynamischen.

Die Qualität und das Ergebnis menschlicher Interaktion hängt nach Edmondson in entscheidender Weise davon ab, wie sicher sich Menschen in dem aktuellen Gruppensetting fühlen. Je sicherer sie sich fühlen, umso eher trauen sich die Gruppenmitglieder Fragen zu stellen, Schwächen und Fehler zuzugeben, Ideen einzubringen sowie den Status quo zu hinterfragen. Unter diesen Umständen wird Lernen möglich, der nach Agyris und Schön als einen Prozess des Erkundens und der Fehlerkorrektur verstanden wird (vgl. Argyris et al. 1978).

"Psychological safety is a sense of confidence that the team will not embarrass, reject, or punish someone for speaking up» (Zitat: Amy Edmondson 1999, S. 354).

Wo wenig psychologische Sicherheit herrscht, verhalten sich Menschen tendenziell wirkungsorientiert, d.h. aus Sorge, einen inkompetenten Eindruck zu machen, werden Ideen nicht genannt, Meinungen zurückgehalten, und eigene Fehler vertuscht. Letztlich zeigen sich darin wiederum die Auswirkungen der oben beschriebenen Lernangst. Anders formuliert tendiert die Gruppe zu einem sozialen Konformismus, also der Tendenz, dass sich die Gruppenmitglieder so verhalten, wie sie glauben, dass es von ihnen erwartet wird. Kreativität und kreative Zerstörung wird so nicht möglich.

Anders erlebt ein Team mit psychologischer Sicherheit einen ständigen „Brainstorming-Zustand». Es entsteht ein positiver Feedbackzirkel, in dem neue Ideen weitere neue Ideen generieren. Ein Zustand, der vor allem in der Phase der Initiierung von Innovationen als erstrebenswert erscheint.

Psychologische Sicherheit beinhaltet zwischenmenschliches Vertrauen. Psychoanalytiker wie Erik Erikson haben das (Ur) Vertrauen entwicklungspsychologisch begründet und festgestellt, dass Menschen ein lebenslanges Vertrauen i. S. eines Gefühls der Verlässlichkeit ihrer Um- und Mitwelt besitzen, weil sie in den ersten Lebensjahren eine stabile und liebevolle Mutterbeziehung erfahren konnten (vgl. Erikson, 
1957). Dies vorausgesetzt wird Vertrauen allgemein häufig als eine Eintrittswahrscheinlichkeit verstanden, mit der eine andere Person ein bestimmtes Verhalten, ein Versprechen oder eine getroffene Abmachung einhält (vgl. Gambetta 1988; Aiken 1993).

In diesem Sinne entwickelt sich Vertrauen aus Vorhersagbarkeit von Verhalten, nicht mehr und nicht weniger. Es sagt nichts darüber aus, inwiefern das eigene Verhalten gerade durch die Vorhersagbarkeit der Reaktion beeinflusst wird und dadurch als wesentlich erachtete Aspekte nicht genannt werden. Dahingegen weist das Konzept der psychologischen Sicherheit explizit darauf hin, dass Menschen sich in diesem Kontext trauen, unabhängig von dem sozialen oder hierarchischen Gefüge, ihre Meinungen zu vertreten, auch und vor allem dann, wenn sie der der Mehrheit widerspricht und in eine andere Richtung weist. Wer hat nicht solche Erfahrungen gemacht, dass in Projektsitzungen, Timelines abgeknickt werden, die praktisch keiner wirklich für realistisch hält. Psychologische Sicherheit schafft einen Raum, in dem Kritik geäussert, Fragen gestellt und die Glaubwürdigkeit steigt, wenn Fehler zugegeben werden.

\section{Erläuterungskasten}

Fragebogen zur Bestimmung der psychologischen Sicherheit.

Der Grad der psychologischen Sicherheit innerhalb eines Teams kann gemäss Amy Edmondson anhand sieben Aussagen ermittelt werden. Die Befragten müssen den Aussagen jeweils auf einer siebenstufigen Antwortskala mehr oder weniger stark zustimmen oder sie mehr oder weniger stark ablehnen.

1. Wenn Sie in diesem Team einen Fehler machen, wird dieser häufig gegen Sie gerichtet.

2. Mitglieder dieses Teams können Probleme und schwierige Themen ansprechen.

3. Leute in diesem Team lehnen andere manchmal ab, weil sie anders sind.

4. Es ist sicher, in diesem Team ein Risiko einzugehen.

5. Es ist schwierig andere Mitglieder dieses Teams um Hilfe zu bitten.

6. Niemand in diesem Team würde absichtlich meine Bemühungen untergraben.

7. In Zusammenarbeit mit Mitgliedern dieses Teams werden meine Fähigkeiten und Talente geschätzt und genutzt.

\section{Organisationale Auswirkungen von psychologischer Sicherheit}

Organisationen oder auch Teams, in denen sich psychologische Sicherheit beobachten lässt, weisen bestimmte Merkmale auf: sie verfolgen einen Ansatz der Selbstorganisation, in dem jeder einzelne eine hohe Verantwortlichkeit übernimmt. Die Führungskraft gestaltet den Rahmen, in der solche Interaktionen möglich werden, übernimmt aber nicht die klassische hierarchische Rolle (vgl. Edmondson, 2008, S. 1-10). Auf die Bedeutung von Freiräumen, Vertrauen und Partizipation für die Innovationsfähigkeit wurde bereits vielfach hingewiesen (vgl. Vahs et al. 2015 / Hauschildt et al., 2016).

Wichtig ist an dieser Stelle, dass allein psychologische Sicherheit nicht zu einem produktiven Zustand des Lernens führt, sondern nur dann, wenn sie gekoppelt ist mit einer hohen Bereitschaft der Teammitglieder, Verantwortung zu übernehmen. Ansonsten bleibt die Gruppe in der sogenannten Komfortzone stecken, in der sich die Gruppenmitglieder zwar sicher fühlen, aber wenig Kreativität und Eigenengagement zeigen (siehe Abbildung 1).

\section{Erläuterungskasten}

- Apathiezone: Mitarbeiter neigen dazu, sich apathisch zu verhalten und verbringen primär ihre Zeit damit, um ihre Position zu kämpfen. Typische Organisationen in diesem Quadranten sind grosse, kopflastige Bürokratien, wo Menschen ihre Funktionen erfüllen. Der bevorzugte Modus ist Dienst nach Vorschrift.

- Angstzone: Die Leute fürchten sich Ideen zu äussern, neue Dinge zu probieren oder Kollegen um Hilfe zu fragen.

- Komfortzone: Die Mitarbeiter arbeiten sehr gerne miteinander zusammen, aber fühlen sich nicht besonders herausgefordert und gehen keine Risiken ein

- Lernzone: Hier liegt der Fokus auf der Zusammenarbeit und Lernen mit dem Ziel, Hochleistungsergebnisse zu erzielen.

Stellt sich die Frage, was Teammitglieder dazu bringt, Verantwortung zu übernehmen. Dabei ist es wichtig, die Zirkularität von voneinander beeinflussenden Faktoren zu bedenken und nicht in einfachen Ursachen-Wirkungszusammenhängen zu verfallen. Je mehr Teammitglieder Verantwortung übernehmen, umso eher ist die Führungsperson bereit, Verantwortung zu delegieren. Je mehr die Führungsperson Verantwortung delegiert, um so grösser ist die Wahrscheinlichkeit, dass Mitarbeitende Verantwortung übernehmen. Auch hier liegt ein gruppendynamischer Prozess zugrunde, der nicht direkt gesteuert werden kann. 
Die zentrale Fragestellung bleibt daher, welche Faktoren, welches Verhalten oder welche Interventionen die Wahrscheinlichkeit erhöhen, dass sich das Phänomen der psychologischen Sicherheit entwickelt und auch bewahrt wird. In hierarchischen Kontexten liegt die Verantwortung beim Teamleiter, Projektleiter oder eben allgemein bei der Führungskraft. Selbstorganisation bedeutet ja in den seltensten Fällen, dass es keine definierte Führungsperson mehr gibt, sondern dass im Team Strukturen und Prozesse gelebt werden, in denen Führungsverantwortung geteilt wahrgenommen wird.

\section{Entwicklung von Psychologischer Sicherheit braucht ein Systemwechsel der Unternehmenskultur}

Psychologische Sicherheit entwickelt sich einerseits durch eine bewusste Gestaltung des sogenannten sozialen Prozesses, was nichts anderes umschreibt als die Gestaltung der Beziehungen zu den einzelnen Teammitgliedern sowie die Gestaltung des gruppendynamischen Prozesses. Anderseits müssen die Grundlagen innerhalb der Unternehmenskultur geschaffen werden, um psychologische Sicherheit überhaupt zu ermöglichen.

Werte und Normen (siehe Abbildung 2) sind Bestandteil der Unternehmenskultur und beinhalten Verhaltensrichtlinien und Verbote, anhand Personen die Angemessenheit von Verhaltensweisen als «richtig» oder «falsch», «gut» oder «schlecht», «schön» oder «hässlich» einstufen können (vgl. Hauschildt et al., 2016). Traditionelle Werte wie Disziplin, Gehorsam, Hierarchie und Macht werden zunehmend von neuen Werten wie Selbstbestimmung, Partizipation, Vertrauen, Kreativität und Freiheit abgelöst und bestimmen die Organisationskultur massgeblich mit.

Wenn ein Unternehmen die Innovationsfähigkeit entwickeln möchte, dann ist es wichtig, geeignete Voraussetzungen $\mathrm{zu}$ schaffen um das Leistungspotenzial der Mitarbeiter umfassend nutzen zu können. Gerade in der Innovation sind Freiräume, Vertrauen und Partizipation besonders wichtig, um die Kreativität und den Unternehmergeist zu fördern (vgl. Vahs et al., 2015, S.199 / Hauschildt et al., 2016, S.105).

Für die Gestaltung der sozialen Prozesse braucht es neben der richtigen Unternehmenskultur auch geeignete Führungskräfte.

- mit ausgeprägten kommunikativen Fähigkeiten

- und Übung in kritischer Selbstreflexion,

- sowie hoher Moderationskompetenzen

- als auch Kenntnissen über entsprechende Tools, wie Retrospektiven, Vorgehen nach der systemischen Schleife, wohlwollendes Hypothetisieren, Kollegiale Beratung, Spiegelungsübungen, analoge Techniken mit Bildern und Metaphern, um das Denken anzuregen, etc.
Aber vor allem braucht es ein entsprechendes Mindset der Führungskräfte. Mindset umfasst dabei bestimmte Grundannahmen und Überzeugungen, die Menschen in ihrem Wahrnehmen und Bewerten von Beobachtetem leiten. Die XY-Theorie von McGregor erweist sich hier als ein anschauliches Beispiel: Wenn eine Führungsperson davon ausgeht, dass der Mensch und damit der Mitarbeiter eher unwillig und faul ist (von McGregor als X-Mensch bezeichnet), wird sie anders führen, als wenn sie die Grundannahme teilt, dass Menschen ein Streben nach Entwicklung haben (Y-Menschen). Wenn Führungskräfte ohne ein überzeugtes Y-Mindset agieren, bleiben die obengenannten Tools Makulatur und können nicht ihre Wirkung entfalten.

Die Paradoxie und zugleich das Dilemma besteht nämlich darin, dass die oben genannten Tools ihre Wirksamkeit nur entfalten, wenn sie mit einer inneren Haltung angeboten werden, der von der Kraft der psychologischen Sicherheit wirklich überzeugt ist und sie nicht als ein Instrument zur Manipulation von Menschen betrachtet. Wenn Menschen den Eindruck haben, dass sie bewusst manipuliert werden, verhalten sie sich wirkungsorientiert (s.o.) und eine Entfaltung des kreativen Potentials wird damit eher verhindert als gefördert. Erst wenn die psychologische Sicherheit fester Bestandteil einer Unternehmenskultur sowie der direkten und indirekten Führung geworden ist, entfaltet sie ihre Wirkung.

\section{Fazit}

Die Innovationsfähigkeit einer Unternehmung kann nicht nur an der Innovationsstrategie oder an den sichtbaren im Markt etablierten Innovationen gemessen werden. Sie wird massgeblich durch die Unternehmenskultur und die Zusammenarbeit der einzelnen Mitarbeiter bestimmt. Denn erst wenn sich die Mitarbeiter in der Lernzone befinden und sich trauen bestehendes zu hinterfragen, kann neues entstehen (siehe Abbildung 1).

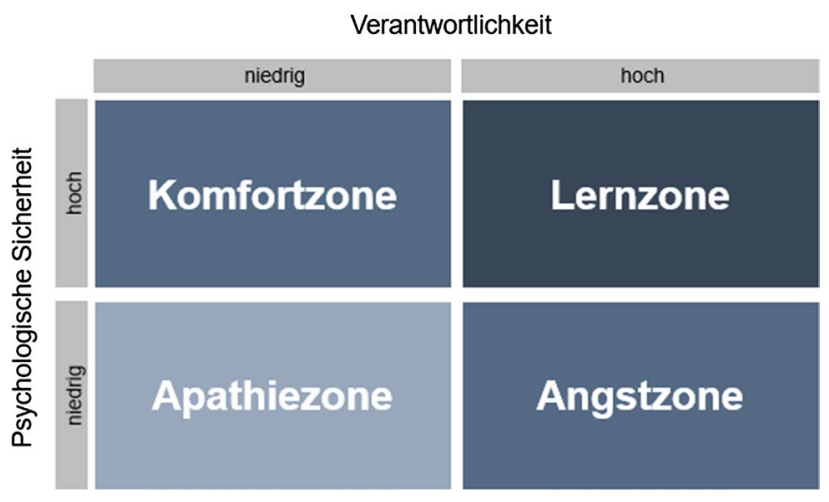

Abbildung 1 Zusammenspiel zwischen psychologischer Sicherheit und Verantwortlichkeit (nach Edmondson, 2008) 


\section{Artefakte \& Symbole}

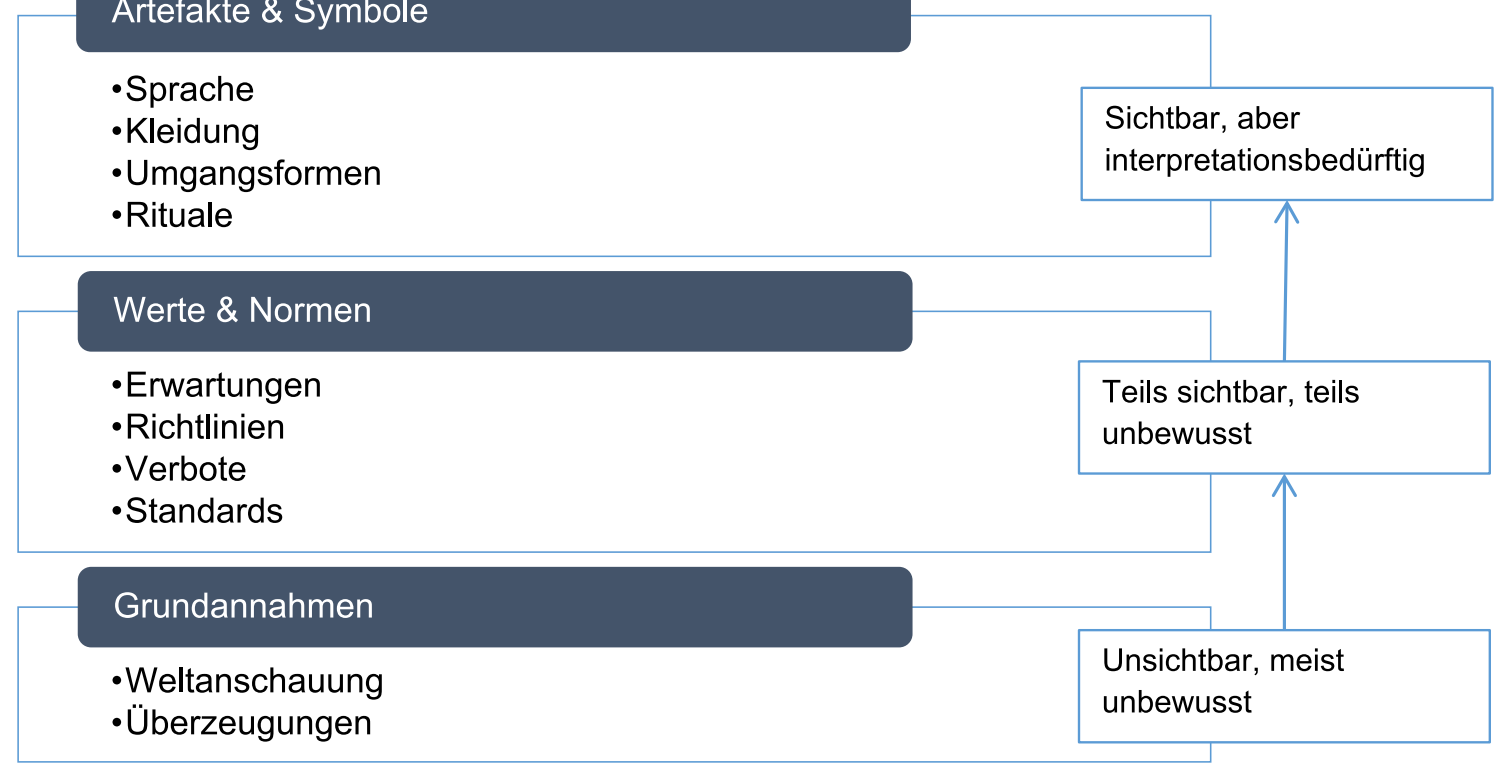

Abbildung 2 Werte und Normen (Eigene Grafik in Anlehnung an Schein, 1984)

\section{Impulse für die Praxis}

- Psychologische Sicherheit fördert Kreativität und Innovationsfähigkeit in Organisationen. Die Etablierung von psychologischer Sicherheit muss einerseits durch die Unterstützung der Führungsperson erfolgen. Durch eine bewusste Gestaltung des sozialen Prozesses mit den Mitarbeitenden wird ein Klima geschaffen, in dem alle Beteiligten ihre Überzeugungen vertreten und Fehler und Schwächen zugegeben können. Andererseits müssen passende Werte im Unternehmen gelebt werden.

- Die Herausforderung besteht in der Bewältigung des Paradox: Werden die Massnahmen und das persönliche Verhalten aus einem instrumentellen Verständnis durchgeführt, mit dem die Menschen zielgerichtet manipuliert werden sollen, entsteht genau das Gegenteil von psychologischer Sicherheit. Nur mit einem entsprechenden Mindset können die Massnahmen, Tools und das persönliche Verhalten dazu beitragen, dass sich Menschen wirklich psychologisch sicher fühlen.

- Psychologische Sicherheit allein führt noch zu keiner erhöhten Innovationsfähigkeit. Gleichermassen braucht es Strukturen, die eine wirkliche Verantwortungsübernahme der einzelnen Teammitglieder ermöglichen und auch einfordern und so eine Lernzone für alle Beteiligten schaffen.

- Psychologische Sicherheit zu etablieren, ist ein kontinuierlicher Prozess, der nie als abgeschlossen oder als sicher gelten kann. Alle Interakteure müssen letztlich durch ihr Handeln immer wieder die Etablierung der psychologischen Sicherheit gegenseitig bestätigen, Irritationen müssen thematisiert werden (Abbildung 2).

Open Access Dieser Artikel wird unter der Creative Commons Namensnennung 4.0 International Lizenz veröffentlicht, welche die Nutzung, Vervielfältigung, Bearbeitung, Verbreitung und Wiedergabe in jeglichem Medium und Format erlaubt, sofern Sie den/die ursprünglichen Autor(en) und die Quelle ordnungsgemäß nennen, einen Link zur Creative Commons Lizenz beifügen und angeben, ob Änderungen vorgenommen wurden. Die in diesem Artikel enthaltenen Bilder und sonstiges Drittmaterial unterliegen ebenfalls der genannten Creative Commons Lizenz, sofern sich aus der Abbildungslegende nichts anderes ergibt. Sofern das betreffende Material nicht unter der genannten Creative Commons Lizenz steht und die betreffende Handlung nicht nach gesetzlichen Vorschriften erlaubt ist, ist für die oben aufgeführten Weiterverwendungen des Materials die Einwilligung des jeweiligen Rechteinhabers einzuholen. Weitere Details zur Lizenz entnehmen Sie bitte der Lizenzinformation auf http://creativecommons.org/licenses/ by/4.0/deed.de.

\section{Literaturverzeichnis}

Aiken LR (1993) Personality: theories, research, and applications. Prentice-Hall College, New Jersey

Argyris C, Schön DA (1978) Organizational learning: a theory of action perspective, reading

Chesbrough H (2006) Open innovation-the new imperative for creating and profiting from technology, p. 31

Cooper RG (2009) How companies are reinventing their idea-to-launch methodologies. Res Technol Manag 5(2):47-57

Edmondson AC (1999) Psychological Safety and Learning Behavior in Work Teams. Admim Sci Q 44(2):350-383 
Edmondson AC (2008) The competitive imperative of learning. Harvard Business Review, Juli-August

Erikson EH (1957) Kindheit und Gesellschaft, 1957, Stuttgart (4. Aufl.)

Gambetta D (1988) Trust: making and breaking cooperative relations. Basil Blackwell, Oxford

Goffin K, Mitchell R (2010) Innovation management—effective strategy and implementation using the Pentathlon Framework, 3rd edn. Macmillan International Higher Education, New York

New York Times Magazine (2016) What Google learned from its quest to build the perfect team, pp 20

Hauschildt J, Salomo J, Schultz C, Kock A (2016) Innovationsmanagement, 6 Auflage. Vahlen, Munich
Kels P, Baumann S, Kocher P-Y (2019) Die erneuerungsfähige Organisation. 02/2019 (88. Jg.), Seite 125-129, 2019

Meyer A, Wrba M, Bachmann T (2018) Psychologische Sicherheit: Das Fundament gelingender Arbeit im Team. Mensch und Computer Usability Professionals, 02.-05. September, Seite 194

Raabe J (2012) Erfolgsfaktoren für Innovation in Unternehmen. Springer, Wiesbaden

Schein EH (1984) Coming to a new awareness of organizational culture. Sloan Manag Rev 25:2 (Page 3)

Vahs D, Brem A (2015) Von der Idee zur erfolgreichen Vermarktung, 5 Auflage. Schäffer-Poeschel, Stuttgart 